\title{
Production and evaluation of guidelines for the management of inflammatory bowel disease: the Leicester experience
}

\author{
A M Read, M A Stone, B J Rathbone, J de Caestecker, A C B Wicks, S Longworth, \\ C R O'Shea, R Baker, D Spence, A W Hall, M J Kelly, J Cannon, R J Robinson, A R Hart, \\ P Miles, J F Mayberry
}

\begin{abstract}
Summary
Consensus guidelines for the management of patients with inflammatory bowel disease were produced by gastroenterologists, gastrointestinal surgeons and a cross-section of general practitioners (GPs) from Leicestershire in order to develop a seamless pattern of care with a common approach to diagnosis and treatment. It was hoped that the guidelines would encourage a movement towards care in the community for many patients with stable disease and so speed up new consultation rates. The study then assessed the impact of these guidelines on the referral letters of GPs to hospital consultants, the prediction of disease and adherence to them on re-referring patients after discharge. The guidelines were distributed to all 487 GPs in the Leicester Health Authority area and the gastroenterology teams within the hospitals. The value of the guidelines was assessed by an audit of referral letters, the length of time from referral letter to out-patient appointment, both before and after the launch of the guidelines, adherence to the guidelines on re-referral, and monitoring the outcome of the discharged patients. Whilst the guidelines may have helped GPs to manage stable patients in the community, the content of referral letters and the diagnostic abilities of GPs were not seen to improve since the launch of the guidelines. However, only $5 \%$ of stable patients who were discharged from one clinic were re-referred for inflammatory bowel disease.
\end{abstract}

Keywords: guidelines; audit; inflammatory bowel disease

During the last 5 years, there has been a marked drive towards the development of guidelines for the management of a number of conditions and gastroenterology has been no exception to this approach to clinical care. General concerns about the need for authoritative statements on diagnosis and management recently led the British Society of Gastroenterology to sponsor such guidelines for a number of conditions including inflammatory bowel disease (IBD).
Before this national initiative was mooted, several centres with an interest in IBD had developed local guidelines, usually employing consensus rather than an evidence-based methodology. The driving force behind these activities was the administrative need to transfer the long-term care of stable patients to the primary sector. The belief that clinicians from different backgrounds can have a common approach to diagnosis and treatment has helped foster this view, which also has a particular appeal to healthcare administrators. These beliefs have been strengthened by the emergence of evidence-based guidelines where assessment and treatment can be based on randomised controlled trials with less opportunity for individual clinical variation in management. As such guidelines are likely to have a significant effect on hospital and general practice, it is critical that their introduction is monitored and their value assessed. Ideally, guidelines should reduce morbidity and mortality. However, other outcome measures which can be considered include frequency of hospital attendance, compliance with drug therapy, and frequency of recurrent attacks. ${ }^{2}{ }^{3}$

With these thoughts in mind, a group of clinicians in Leicestershire set up a Guidelines Committee in 1992 to develop a consensus on the management of a number of common gastrointestinal conditions. ${ }^{4}$ The rationale for the group was the devolution of care of stable patients to their family doctors within the structured clinical environment proposed by the guidelines. General practitioners (GPs) want to share the care of gastroenterology patients with hospital consultants with the support of guidelines. ${ }^{5}$ IBD was chosen as the first disease group to be considered because of the infrequency with which it is seen by any one GP, as well as the limited number of drugs available for management. The intention of the committee was to produce a professionally printed document ${ }^{6}$ which would be helpful to individual GPs and to evaluate its benefits at the end of the first year of use.

\section{Methods}

The method used for the production of the guidelines was a series of meetings in which a consensus on diagnosis and management was reached. This led to the subsequent publi- 
cation of a document which was distributed to GPs throughout Leicestershire. The guidelines were then assessed through a series of studies on their impact on clinical practice.

THE GUIDELINES COMMITTEE

The committee was drawn from hospitals in the Leicestershire Health Authority and consisted of all gastroenterologists and surgeons with an interest in gastroenterology. In addition, the committee had GP members, including representatives from the Family Health Service Authority (FHSA), GP fundholders, and non-fund-holding practices, as well as a member of the Eli Lilly National Clinical Audit Centre. Other members of the committee included a pharmacist, a hospital management representative, a nurse, and two junior doctors. In addition to her professional input, the nurse acted as Project Facilitator. This involved co-ordination of the committee, implementation of its proposals, and their subsequent evaluation.

The committee met for an hour a month. Following these meetings, the draft consensus document was revised by members of the group, distributed widely for critical comment and reconsidered at subsequent meetings. A near-final version was submitted to the FHSA and to the Local Medical Committee for comments. These were incorporated into the document and the committee then finalised the guidelines which were professionally printed.

\section{PATIENT PARTICIPATION}

Due to some anxieties on the part of the Guidelines Committee, patients' opinions on shared care were sought before distribution. A small sample of 17 consecutive patients attending two IBD clinics in Leicestershire were asked their views on this approach using a structured questionnaire completed by the Project Facilitator.

\section{DISTRIBUTION OF GUIDELINES}

In total, 487 sets of guidelines were distributed to every GP in the 150 practices of Leicestershire and to relevant wards and hospital doctors in Leicestershire. This initial launch was supported by a series of meetings with GPs in community hospitals and health centres. Each meeting had postgraduate educational approval. Both the Guidelines and these supporting meetings received publicity in the local press and national medical newspapers. As part of a programme of on-going support throughout the first year of the guidelines, a mobile phone number was made available to GPs and practice nurses for easy access to specialist advice or to discuss management problems. When patients attended out-patient clinics, a smaller version of the guidelines was sent out with their letter, designed to be put into the patient's GP's notes.

ASSESSMENT AND IMPACT ON CLINICAL PRACTICE The impact of guidelines was assessed using the following criteria:
- content of referral letters (eg, details of foreign travel, smoking, family history of IBD)

- time between first referral and first consultation

- ability of GPs to predict diagnosis

- unnecessary repetition of tests performed in general practice by hospital doctors.

The guidelines included areas which could influence clinical activity and outcome (process and outcome audit).

Two months after launch the senior partners of half the practices in Leicestershire were contacted. They were asked whether they had received the guidelines, whether they had found them of value, and how had they made use of them.

During a 24-month period, one of four IBD clinics in Leicestershire was monitored and patients who were discharged with stable IBD were recorded. Patients' case notes were reviewed during the study period to see if they had been re-referred for an IBD-related problem. These re-referrals were compared to the options given within the guidelines for re-referral to hospital care.

\section{Results}

PATIENTS' VIEWS ON SHARED CARE

Before the distribution of the guidelines, 12 of 17 patients $(71 \%)$ said they would be happy to be looked after entirely by their GP with support from a hospital doctor. However, eight of them $(47 \%)$ had concerns about their GP's knowledge of the disease and 16 (94\%) patients favoured a co-operative care card for use by GPs and hospital doctors. This would record the extent of disease and current treatment. Fifteen $(88 \%)$ patients wanted more information leaflets in hospital out-patient departments and $12(70 \%)$ wanted them available in general practices.

\section{GP SURVEY ON GUIDELINE USE}

Almost half the GP practices in Leicester $(72 / 150)$ were contacted in a post-launch survey and replies were received from 45 (response rate $60 \%$ ). Thirty-six of these 45 GPs $(80 \%)$ recalled having received the guidelines and 32 described them as useful. Nine respondents $(20 \%)$ had actually used them during a consultation.

SURVEY OF GPS ONE YEAR AFTER THE

INTRODUCTION OF GUIDELINES

Of the 487 GPs in Leicestershire, 106 (21\%) sent replies to the first mailing about their use of guidelines in the preceding year and 52 (total $32 \%$ ) from a reminder mailing. Although no data are available from non-respondents, it seems likely that they had only limited interest in the guidelines. Of the 158 GPs responding, $68(43 \%)$ had used the guidelines when referring a patient to a hospital specialist and 49 $(31 \%)$ during an actual consultation; this rate had remained unchanged during the year. A third of the GPs (52) had made use of the guidelines when reaching a diagnosis and 15 (9\%) during GP training. Eight GPs (5\%) had 
Table 1 Effect of guidelines on the content of a sample of referral letters

\begin{tabular}{lll}
\hline & $\begin{array}{l}\text { Before guidelines } \\
(n=20)\end{array}$ & $\begin{array}{l}\text { After guidelines } \\
(n=18)\end{array}$ \\
\hline $\begin{array}{l}\text { Recorded history } \\
\text { Travel }\end{array}$ & 3 & 3 \\
Smoking & 1 & 0 \\
Family history & 0 & 1 \\
Tests performed & & \\
Platelet count & 7 & 6 \\
Plasma viscosity & 1 & 5 \\
Stool culture & 8 & 3 \\
\hline
\end{tabular}

used them during sessions with district and practice nurses and two (1.5\%) with health visitors. General comments about the guidelines included the need for regular updates, their availability on computer disc, and the need for a hospital-based telephone advice line. However, the mobile telephone support which was available to GPs was never used during the study.

ASSESSMENT AND IMPACT ON CLINICAL PRACTICE The guidelines had no significant effect on the content of referral letters, with few details of foreign travel, smoking history or family history being recorded (table 1). One aim of the guidelines was to reduce the period between initial referral and first consultation in outpatients. For the 32 patients studied in the month prior to issuing guidelines, the average time interval was 1.2 months (range 1-4 months). When the study was repeated some 2 months following their issue, the average duration was not significantly different at 1.6 months (range 1-4 months).

The diagnostic accuracy in patients with no previous history of inflammatory bowel disease by GPs was also investigated in one of the four specialist IBD clinics in Leicestershire (table 2). There was no improvement by GPs in their ability to identify new patients with either ulcerative colitis or Crohn's disease.

A further aim of these guidelines was to reduce the unnecessary repetition of tests by hospital doctors. In practice, there was little effect on the repetition of platelet counts, plasma viscosity or stool cultures taken by GPs. In the period prior to the guidelines, eight out of 25 patients referred by GPs had tests, at least one of which was to be subsequently monitored. In three $(37.5 \%)$ cases these were repeated by hospital doctors. In the period after the issuing of guidelines, seven out of 18 patients $(38 \%)$ had tests reported and in all cases these were repeated by hospital doctors.

Table 2 Diagnostic acumen of GPs before and after the issue of guidelines

\begin{tabular}{lll}
\hline & $\begin{array}{l}\text { Number of possible } \\
\text { cases of IBD }\end{array}$ & $\begin{array}{l}\text { Symptoms of IBD but } \\
\text { diagnosis not suggested }\end{array}$ \\
\hline $\begin{array}{l}\text { Before guidelines } \\
\text { Diagnosis or symptoms suggested in referral letter }\end{array}$ & 20 & 25 \\
$\begin{array}{l}\text { Confirmed by investigations in hospital } \\
\text { After guidelines }\end{array}$ & 3 & 6 \\
$\begin{array}{l}\text { Diagnosis or symptoms suggested in referral letter } \\
\text { Confirmed by investigations in hospital }\end{array}$ & 5 & 13 \\
\hline
\end{tabular}

Thus, the rate of testing by GPs did not increase after issue of the guidelines $(z=-0.47$, not significant), but the rate of repetition of tests by hospital doctors did $(\mathrm{z}=2.56$, $\mathrm{p}<0.01)$.

In contrast, guidelines proved effective in helping GPs decide when patients should be re-referred to the hospital service. In total, 56 patients with stable IBD were discharged from the one clinic studied. They were monitored by consulting case notes throughout a 2-year period. Of these, three (5\%) were re-referred with an IBD-related problem and in all cases the referral was within the guidelines for making such a decision. Reasons included a poor response to steroid use and symptoms that were causing concern.

\section{Discussion}

Guidelines can help establish a framework within which those less familiar with certain diseases are able to safely initiate treatment and provide on-going care. They should promote "effective health care by reinforcing good clinical practice and promote change in professionals". ${ }^{3}$ As the responsibility for chronic care is shifted from the secondary sector back towards family practice, the need for guidelines has become even greater. In an ideal world such advice should be evidence-based, but when this is unavailable a consensus view on diagnosis and management can be valuable, although it may not produce the best answers. On the beneficial side, such an approach can also help disseminate expertise widely through the medical community. These types of benefit are being increasingly recognised and in Italy have led to a computerised teaching and medical decision support system.

The need for such an approach was recognised early in the 1990s in Leicestershire and had led directly to the development of a Guidelines Group in Gastroenterology. Its method of working has already been described in detail, ${ }^{4}$ but essentially the group approached their task with an open mind. Their only restrictions were self-imposed and required that any guidelines should be short, easy to read, and of practical value, giving clear advice on areas of controversy for the non-specialist. As a result, the guidelines can sometimes be viewed as didactic. To limit this effect it is important that the target audience feels it has been part of the process and so has some ownership of the document.

Evaluation of the effectiveness of guidelines is important in the face of the widely held belief that they probably do not change practice. The evaluation needs to be rigorous and four models have been suggested:

- randomised trials of patients: doctors are contaminated by their knowledge of the guidelines and so there is no true control group

- randomised trials of doctors: the chosen doctors may feel favoured and so act differently

- controlled before-and-after studies

- balanced incomplete block designs in which doctors from different geographical areas are 
targeted. All doctors are working with guidelines but for different conditions. Comparisons are made between the areas for the effect of guidelines or their absence on the management of these distinct diseases.

This project falls into the "before-and-after" model. Its purpose was to test the mechanism for producing guidelines, their acceptability to both hospital doctors and GPs, and to assess their effectiveness. The practical process of reaching a consensus and ensuring ownership of the guidelines was time consuming and showed the difficulties reaching a common view on management. The failure of the guidelines to reduce the rate of inappropriate tests initiated by hospital doctors (indeed their introduction seems to have stimulated this

1 Anon. Inflammatory bowel disease: Guidelines in gastroenterolgy, No 4. London: British Society of Gastroenterology, ogy, No.

2 Grimshaw J, Russell I. Effect of clinical guidelines on medical practice: a systematic review of rigorous evaluation. Lancal practice: a systematic
cet 1993;322:1317-22.

3 Eccles M, Grimshaw J. The development and implementations of clinical guidelines. London: Royal College of General Practitioners, 1994; report no 26.

4 Read A, Mayberry JF. Inflammatory bowel disease. Developing local management guidelines. Smith Kline Beecham Pharmaceuticals. Mundells ISBN 0948271248: 1996. practice), raises questions as to the effectiveness with which they were introduced into the hospital out-patient environment and to junior doctors in particular. The results are mixed and strongly emphasise the need for on-going education of doctors to encourage them to work within guidelines if they are to be effective. However, the success of the approach was seen in the continued use of guidelines by at least a third of GPs in Leicester over the year, and in their application when re-referral of patients was needed.

We would like to thank Gastrointestinal Education And Research (GEAR) for its support of the Project Facilitator during this study.

5 Moody GA, Mann R, Gay S, Wicks ACB, Mayberry JF. The gastroenterology service: a survey of general practitioners requirements. FR Soc Med 1993;86:26-7.

6 Anon. Inflammatory bowel disease: guidelines for general practitioner and hospital doctors in Leicestershire. Gastroenterology Research Unit. Leicester. ISBN 1898173990: 1994.

7 Ricci E, Rocca G, Giura V, et al. Computerised clinical guidelines in inflammatory bowel disease (IBD). Gut 1996; 39 (suppl 3):A240:1466. 